\title{
Analysis of lung function in a Colombian military with a medical history of thoracic trauma
}

\author{
Alirio Bastidas-Goyes ${ }^{1 *}$, Gustavo Hincapié-Díaz ${ }^{2}$, Eduardo Tuta-Quintero ${ }^{1}$, and Sandra Rodríguez-Rojas ${ }^{2}$ \\ ${ }^{1}$ Departament of Internal Medicine, Universidad de La Sabana, Chía; ${ }^{2}$ Service of Pulmonology, Hospital Militar Central. Bogotá, Colombia
}

\begin{abstract}
Introduction: Pulmonary function can be affected months after blunt thoracic trauma. However, the data reported are scarce. Objective: The objective of the study was to evaluate pulmonary function at 3 and 6 months in subjects with thoracic injuries suffered in combat. Materials and methods: Study of cases and controls evaluating subjects with chest trauma at 3 and 6 months after it, the controls were healthy soldiers with combat experience. Spirometric variables, lung volumes, Cooper test, 6 min walking test, and cardiopulmonary exercise test were evaluated between 2011 and 2016. Results: Forty-five male subjects entered the study, 25 cases evaluated 3 months and 11 at 6 months after the trauma, compared with 20 controls. After 3 months of evaluation, the subjects wounded in combat walked $43.63 \mathrm{~m}(p=0.007)$ less when compared to the control group in the 6 min walk and the post-vital capacity was 0.88 I $(p<0.001)$ lower at 6 months than in the control group. When comparing the subjects with chest trauma evaluated, the only variables that showed a statistically significant recovery were the pre-forced expiratory flow $(p=0.005)$ and 6 min walking test $(p=0.002)$. Conclusion: Subjects with chest trauma in combat show a decrease in lung volumes and a cardiopulmonary exercise test at 3 and 6 months of evaluation.
\end{abstract}

Key words: Respiratory function tests. 6 min walking test. War-related injuries. Combat-related disorders.

\section{Introduction}

The history of humanity has been marked by multiple armed conflicts over the centuries, generating an annual incidence of blunt traumatic lung injuries due to war of $8-10 \%{ }^{1}$. It is estimated that more than $50 \%$ of the anatomical distribution of combat injuries are in the thoracic region, generating mainly pulmonary contusions and pneumothorax ${ }^{2}$. Chest trauma and its complications are the third leading cause of death in all age groups worldwide after cardiovascular diseases and cancer ${ }^{3}$; mortality ranges between 4 and $25 \%$ depending on age, comorbidities, and type of injury. However, the worst outcomes are associated with thoracic vascular trauma or flail chest ${ }^{4}$. Chest trauma can be divided into two types: non-penetrating and penetrating, the latter accounting for $20 \%$ of all chest trauma ${ }^{3,4}$.

Penetrating gunshot wounds generate injury from direct tissue disruption by projectiles as well as from the energy waves produced. Furthermore, explosions generate toxic gases that can compromise the airways ${ }^{5}$. Lung injury secondary to trauma triggers a cellular immune response mediated initially by neutrophils-mononuclear cells and an increase in levels of macrophage cyclooxygenase 1 , interleukin 6 , and myeloperoxidase, among others ${ }^{6}$. This can lead to local injury with the development of acute respiratory distress syndrome (ARDS) and systemic involvement ${ }^{7,8}$. Patients who manage to survive acute trauma due to parenchymal
Correspondence:

*Alirio Bastidas-Goyes

E-mail: alirio.bastidas@unisabana.edu.co

0185-1063/@ 2021 Sociedad Médica del Hospital General de Mexico. Published by Permanyer. This is an open access article under the CC BY-
NC-ND license (http://creativecommons.org/licenses/by-nc-nd/4.0/).
Date of reception: 24-01-2021

Date of acceptance: 06-05-2021
Available online: 29-07-2021

Rev Med Hosp Gen Mex. 2021;84(3):110-115 www.hospitalgeneral.mx 
involvement with decreased pulmonary compliance and generation of toxic gases during the explosions may manifest with restrictive and obstructive alterations ${ }^{7,9}$.

The body's ability to tolerate exercise depends on proper integration of the respiratory, cardiovascular, and musculoskeletal systems. Pulmonary function tests (PFTs) have provided guidance on the origin of the patient's functional pathology, whether obstructive or restrictive, playing a key role in the diagnosis and follow-up of respiratory disease in patients with chest trauma, complications such as ARDS and surgical outcomes ${ }^{10,11}$. Moreover, the 6 min walk distance (6MWD) can decrease in $72 \%$ of chest trauma patients up to 6 months post-injury ${ }^{12}$. Hence, a diagnostic approach to symptoms reported by patients with penetrating chest injuries and $\mathrm{PFT}^{13}$ can provide guidance on the patient's condition and associated complications in war veterans.

Patients with a history of chest trauma may be affected in their respiratory function. However, data on pulmonary function alterations in subjects with chest trauma secondary to combat injuries are scarce $\mathrm{e}^{11-13}$. The aim of the following study is to measure with incremental and non-incremental PFT, the exercise limitation of combat-wounded military personnel with chest injuries.

\section{Material and methods}

A case-control study was conducted in the pulmonology department of Hospital Militar Central (Bogotá, Colombia), assessing subjects with a history of chest trauma at 3 and 6 months. Spirometric variables, lung volumes, Cooper test, 6MW, and cardiopulmonary exercise test (CPET) were evaluated between 2011 and 2016.

\section{Eligibility criteria}

The military population over 18 years of age with chest trauma who underwent medical pain management or surgery (thoracotomy or laparotomy) was included in the study. The population with a history of vascular, otic or ocular trauma, cranioencephalic, spinal, abdominal trauma, and traumatic amputation of any limb was excluded from the study.

\section{PFT, 6MW, Cooper test, and CPET}

Study subjects underwent pre- and post-beta2-agonist spirometry with measurement of forced vital capacity $(F V C)$, forced expiratory volume in the $1^{\text {st }} \mathrm{s}$ (FEV1), peak expiratory flow (PEF), total lung capacity (TLC), and vital capacity (VC). In addition to diffusing capacity for carbon monoxide (DLCO), integrated CPET with measurement of oxygen consumption (VO2) and oxygen consumption per kilogram of body weight (VO2/kg) according to the recommendations of the American Thoracic Society (ATS) and European Respiratory Society $\left(E R S^{10,11}\right.$. The $6 \mathrm{MW}$ was performed by measuring the distance covered for $6 \mathrm{~min}$ in a $30 \mathrm{~m}$ course ${ }^{12}$. The Cooper test was performed by measuring the distance covered as far as possible in 12 min with an active, steady jog without pause, according to the physical possibilities of each person. Pursuant to the distance covered, aerobic capacity was categorized as very poor, poor, fair, good, and excellent ${ }^{13}$.

\section{Variables}

For both groups, information on birthdate, weight, height, body mass index (BMI), military rank, and educational level was obtained. Weapon type, Injury Severity Score (ISS), days in hospital or intensive care unit (ICU), health care-associated infection, and injured structure were determined for the case group. Sociodemographic and clinical data were obtained from queries and patient records.

The sample size was calculated using the Epidat 4.0 software considering a standardized mean difference of 0.9 in the PFT of two independent groups, power of $80 \%$ and confidence level of $95 \%$, a minimum of 21 subjects in each study group.

\section{Statistical analysis}

A descriptive analysis was performed summarizing quantitative variables in averages and standard deviations if the distribution was normal, as well as median and interquartile ranges, if it was not normal, qualitative variables in frequencies and percentages. Quantitative variables were compared using Student's t-test or Mann-Whitney U-test depending on their distribution, qualitative variables were compared using the Chisquare test, with $p<0.05$ being considered statistically significant. Statistical analysis was carried out using SPSS version 25 licensed software.

\section{Ethical considerations}

The study protocol was approved by the research ethics committee of Hospital Militar Central (protocol record C-06-2011), with informed consent signed by all study subjects. 
Table 1. Baseline characteristics of the study population

\begin{tabular}{|c|c|c|c|c|}
\hline Variable & Total population $(n=45)$ & Wounded in action $(n=25)$ & Healthy subjects $(n=20)$ & p-value \\
\hline Mean age (SD) & $28(7.22)$ & $24.1(4.99)$ & $33(6.75)$ & $<0.001$ \\
\hline Mean height (SD) & 170.33 (5.38) & $170.4(6.67)$ & $170.2(4.7)$ & 0.893 \\
\hline Mean weight (SD) & $69.7(11.5)$ & $63.89(8.81)$ & $77.1(10.2)$ & $<0.001$ \\
\hline Mean BMI, kg/m² (SD) & $24.08(4.03)$ & $22.03(3.18)$ & $26.64(3.5)$ & $<0.001$ \\
\hline $\begin{array}{l}\text { Military rank, n (\%) } \\
\text { Soldier } \\
\text { Warrant officer } \\
\text { Officer }\end{array}$ & $\begin{array}{c}25(55.6) \\
15(33.3) \\
5(11.1)\end{array}$ & $\begin{array}{c}20(80) \\
3(12) \\
2(8)\end{array}$ & $\begin{array}{c}5(25) \\
12(60) \\
3(15)\end{array}$ & $\begin{array}{l}<0.001 \\
<0.001 \\
<0.001\end{array}$ \\
\hline $\begin{array}{l}\text { Level of education, } \mathrm{n}(\% \\
\text { Primary } \\
\text { Baccalaureate } \\
\text { Technician } \\
\text { University } \\
\text { Pain, } \mathrm{n}(\%) \\
\text { Dyspnea, } \mathrm{n}(\%)\end{array}$ & $\begin{array}{c}12(26.7) \\
18(40) \\
6(13.3) \\
9(20) \\
23(51) \\
18(40)\end{array}$ & $\begin{array}{c}12(48) \\
6(24) \\
4(16) \\
3(12) \\
22(88) \\
18(72)\end{array}$ & $\begin{array}{c}0(0) \\
12(60) \\
2(10) \\
6(30) \\
1(5) \\
0(0)\end{array}$ & $\begin{array}{c}0.019 \\
0.019 \\
0.019 \\
0.019 \\
<0.001 \\
<0.001\end{array}$ \\
\hline
\end{tabular}

BMI: body mass index; HVGI: high-velocity gunshot injury; kg: kilogram; LVGI: low-velocity gunshot injury; m: mean; m2: square meter; n: number; SD: standard deviation.

\section{Results}

Forty-five male subjects entered the study, 25 cases evaluated at 3 months and 11 cases at 6 months after trauma, compared to 20 controls. In combat-wounded subjects, the mean age was 24.1 years (SD: 4.99 ), $80 \%$ $(20 / 25)(p<0.001)$ were soldiers and only $12 \%(3 / 25)$ $(p<0.019)$ had a university degree (Table 1). About $88 \%(22 / 25)(p<0.001)$ of the subjects with combat-related chest injuries reported pain and only $5 \%(1 / 20)$ $(p<0.001)$ of the controls reported this symptom.

Hypervelocity projectiles caused $76 \%$ of all combat-related injuries. The average number of days in hospital was 17.5 (SD: 16.2$), 56 \%$ (14/25) were admitted to the ICU with an average duration of 7.2 (SD: 5.54 ) days. About $92 \%(23 / 25)$ of subjects with a history of trauma required surgical management and 64\% (16/25) had chest wall injuries. About $8 \%(2 / 25)$ of subjects with combat-related chest injuries developed ARDS. About 64\% (16/25) of subjects with combat-related chest injuries were in the health battalion as a site of post-hospitalization care. The population characteristics are shown in table 2 .

\section{Comparison of pulmonary function between controls and trauma subjects at 3 months of assessment}

A decrease in PFT measured in the subjects with a history of chest trauma was recorded at 3 months of assessment, compared to the control group in table 3 .
Table 2. Characteristics of severity and thoracic involvement in combat-wounded subjects

\begin{tabular}{|l|c|}
\hline Variable & $\mathbf{n}(=25)$ \\
\hline Type of weapon, $\mathrm{n}$ & \\
LVGI & $1(4)$ \\
HVGI & $19(76)$ \\
Explosive agent & $2(8)$ \\
Projectile and explosive agent & $3(12)$ \\
Mean ISS (SD) & $6.9(3.50)$ \\
Mean inpatient stay (SD) & $17.5(16.2)$ \\
ICU days, $\mathrm{n}(\%)$ & $14(56)$ \\
Mean ICU stay (SD) & $7.2(5.54)$ \\
Pneumonia, $\mathrm{n}(\%)$ & $8(32)$ \\
Catheter-associated infection, $\mathrm{n}(\%)$ & $21(84)$ \\
Surgical management, $\mathrm{n}(\%)$ & $23(92)$ \\
Thoracotomy, $\mathrm{n}(\%)$ & $20(80)$ \\
Laparotomy, $\mathrm{n}(\%)$ & $9(36)$ \\
\hline Injured structure, $\mathrm{n}(\%)$ & \\
Lung & $12(48)$ \\
Diaphragm & $6(24)$ \\
Chest wall & $16(64)$ \\
Extremities & $12(48)$ \\
\hline Health-care site, $\mathrm{n}(\%)$ & \\
Home & $6(24)$ \\
Health Battalion & $16(64)$ \\
Unit in charge & $3(12)$ \\
\hline
\end{tabular}

HVGI: high-velocity gunshot injury; ICU: intensive care unit; ISS: injury severity score; LVGI: low-velocity gunshot injury; m: mean; n: number; SD: standard deviation.

Combat injured subjects at 3 months of assessment walked $43.63 \mathrm{~m}$ (485.67 vs. 529.30$)(p=0.007)$ less when compared to the control group at the 6MW. DLCO was $11.38 \mathrm{ml} / \mathrm{mmHg} / \mathrm{min}(\mathrm{p}<0.001)$ lower at 3 months of assessment compared to the control group. 
Table 3. Pulmonary function between controls and trauma subjects at 3 months of assessment

\begin{tabular}{|c|c|c|c|c|}
\hline Variables & Total population ( $\mathrm{n}=45$ ) & Valuation 3 months $(n=25)$ & Healthy subjects $(n=20)$ & p-value \\
\hline Cooper test $\mathrm{m}$ (SD) & $1297(574.4)$ & $993(572.43)$ & 1677 (276.73) & $<0.001$ \\
\hline $6 \mathrm{MW} \mathrm{mt} \mathrm{m}$ (SD) & $505.06(105.31)$ & $485.67(104.40)$ & $529.30(103.95)$ & 0.007 \\
\hline Pre-FVC L m (SD) & $5.10(1.21)$ & $4.46(1.13)$ & $5.90(0.74)$ & $<0.001$ \\
\hline Post-FVC L m (SD) & $5.02(1.21)$ & $4.37(1.11)$ & $5.83(0.77)$ & $<0.001$ \\
\hline Pre-FEV1 L m (SD) & $4.27(0.96)$ & $3.8(0.92)$ & $4.86(0.63)$ & $<0.001$ \\
\hline Post-FEV1 L m (SD) & $4.31(0.94)$ & $3.84(0.92)$ & $4.89(0.59)$ & $<0.001$ \\
\hline Pre-FEV1/FVC L m (SD) & $84.33(6.83)$ & $85.76(7.53)$ & $82.55(5.52)$ & 0.018 \\
\hline Post-FEV1/FVC L m (SD) & $85.38(6.66)$ & $87.16(6.91)$ & $83.15(5.74)$ & 0.043 \\
\hline Pre-PEF L m (SD) & $10.26(2.81)$ & $8.46(1.98)$ & $12.51(1.95)$ & $<0.001$ \\
\hline Post-PEF L m (SD) & $11.14(3.20)$ & $9.67(2.76)$ & $12.98(2.77)$ & $<0.001$ \\
\hline Pre-TLC L m (SD) & $6.50(1.34)$ & $5.8(1.17)$ & $7.28(1.15)$ & $<0.001$ \\
\hline Post-TLC L m (SD) & $6.40(1.39)$ & $5.88(1.30)$ & $7.05(1.24)$ & 0.004 \\
\hline Pre-VC L m (SD) & $5.29(1.21)$ & $4.69(1.16)$ & $6.05(0.81)$ & $<0.001$ \\
\hline Post-VC L m (SD) & $5.25(1.23)$ & $4.62(1.17)$ & $6.03(0.79)$ & $<0.001$ \\
\hline $\mathrm{DCLO} \mathrm{mL} / \mathrm{mmHg} / \mathrm{min} \mathrm{m}$ (SD) & $41.60(26.21)$ & $36.54(22.15)$ & $47.92(20.23)$ & $<0.001$ \\
\hline V02 mL/kg/min m (SD) & 36.78 (8.98) & $32.38(7.05)$ & $42.29(8.16)$ & $<0.001$ \\
\hline
\end{tabular}

DCLO: diffusing capacity for carbon monoxide; FEV1: forced expiratory volume in the $1^{\text {st }}$ second; FVC: forced vital capacity; kg: kilogram; L: liter; m: mean; $\mathrm{mL}$ : milliliter; min: minute; mmHg: millimeters of mercury; mt: meters; $\mathrm{n}$ : number

V02: oxygen consumption; PEF: peak expiratory flow; SD: standard deviation; TLC: total lung capacity; VC: vital capacity; $6 \mathrm{MW}$ : 6 min walk.

\section{Comparison of pulmonary function between controls and trauma subjects at 6 months of assessment}

At 6 months, the assessment regarding the 6MW, pre-/post-FVC, pre-/post-VC, and VO2/kg in the subjects with a history of chest trauma remained lower than in the control group with a statistically significant $p$-statistic as per table 3. In combat-wounded subjects, pre-/postVC was $0.88 \mathrm{I}(\mathrm{p}<0.001)$ lower than in the control group. $\mathrm{VO} 2 / \mathrm{kg} / \mathrm{min}$ was $5.28 \mathrm{ml} / \mathrm{kg} / \mathrm{min}(\mathrm{p}=0.002)$ lower at 6 months of assessment than in the control group.

When comparing subjects with chest trauma assessed at 3 and 6 months, the only variables that showed statistically significant recovery were PEF with a recovery of $2.27 \mathrm{I}(\mathrm{p}=0.005)$ and $6 \mathrm{MW}$ with a further $19.93 \mathrm{~m}$ distance covered $(p=0.002)$ (Table 4).

\section{Discussion}

Deterioration of pulmonary function was found in military personnel with a history of chest trauma in combat at 3 and 6 months of assessment. PEF and 6MW were the only variables showing significant change between 3 and 6 months of assessment in subjects with a history of chest trauma. Dyspnea and pain are persistent symptoms in these subjects and physical fitness remains significantly decreased. HVGI was the most frequently encountered condition in these patients as well as catheter-associated infection. Pneumonia and ARDS were the most frequent complications.

Herridge et al. in two cohort studies evaluated 109 survivors of ARDS and chest trauma for 6, 12, and 72 months, showing mild restrictive compromise of pulmonary function in the first 3 months of follow-up reaching normal values at 6 months and a single increase in FVC up to 1 year of assessment ${ }^{14,15}$. The restrictive chest injury reflected by low lung volume measurements can be maintained for a considerable time in patients with chest trauma, and this time may vary due to severity and associated complications. In our study, where chest injuries may have a greater complexity than the type of injury described in these cohort studies, this may influence the restrictive lung dysfunction remaining at 6 months of observation. Our results showed a statistically significant 
Table 4. Pulmonary function between controls and trauma subjects at 6 months of assessment

\begin{tabular}{|l|c|c|c|c|}
\hline Variables & Total population (n= 31) & Valuation $\mathbf{6}$ months $\mathbf{( n = 1 1 )}$ & Healthy subjects $(\mathbf{n}=\mathbf{2 0})$ & $\mathbf{p}$-value \\
\hline Cooper test m (SD) & $1560.5(397.43)$ & $1348.9(501.96)$ & $1677(276.73)$ & 0.008 \\
\hline 6MW mt m (SD) & $515.08(108.2)$ & $501.6(109.52)$ & $529.3(103.95)$ & 0.004 \\
\hline Pre-FVC L m (SD) & $5.57(0.93)$ & $4.99(1.0)$ & $5.90(0.74)$ & 0.002 \\
\hline Post-FVC L m (SD) & $5.53(0.96)$ & $4.98(1.06)$ & $5.83(0.77)$ & 0.004 \\
\hline Pre-FEV1 L m (SD) & $4.65(0.78)$ & $4.29(0.92)$ & $4.86(0.63)$ & 0.008 \\
\hline Post-FEV1 L m (SD) & $4.74(0.75)$ & $4.46(0.94)$ & $4.89(0.59)$ & 0.006 \\
\hline Pre-FEV1/FVC L m (SD) & $83.84(6.71)$ & $86.18(8.23)$ & $82.55(5.52)$ & 0.085 \\
\hline Post-FEV1/FVC L m (SD) & $84.74(6.73)$ & $87.64(7.67)$ & $12.51(1.95)$ & 0.005 \\
\hline Pre-PEF L m (SD) & $11.88(2.54)$ & $10.73(3.13)$ & $12.98(2.77)$ & 0.024 \\
\hline Post-PEF L m (SD) & $12.35(2.96)$ & $11.20(3.09)$ & $7.28(1.15)$ & 0.051 \\
\hline Pre-TLC L m (SD) & $6.96(1.25)$ & $6.39(1.26)$ & $7.05(1.24)$ & 0.019 \\
\hline Post-TLC L m (SD) & $6.69(1.29)$ & $6.03(1.16)$ & $3.84(0.60)$ & 0.007 \\
\hline Pre-VC L m (SD) & $3.55(0.83)$ & $3.03(0.97)$ & $3.82(0.58)$ & $<0.001$ \\
\hline Post-VC L m (SD) & $3.51(0.79)$ & $2.94(0.83)$ & $47.92(20.23)$ & $<0.001$ \\
\hline DCLO mL/mmHg/min m (SD) & $45.62(25.75)$ & $41.43(29.07)$ & $42.29(8.16)$ & 0.018 \\
\hline V02 mL/kg/min m (SD) & $39.91(9.01)$ & $37.01(7.58)$ & 0.002 \\
\hline
\end{tabular}

DCLO: diffusing capacity for carbon monoxide; FEV1: forced expiratory volume in the $1^{\text {st }}$ second; FVC: forced vital capacity; kg: kilogram; L: liter; m: mean; min, minute; mL: milliliter; mmHg: millimeters of mercury; mt: meters; n: number; V02: oxygen consumption; PEF: peak expiratory flow; SD: standard deviation; TLC: total lung capacity; VC: vital capacity; 6MW: 6 min walk.

recovery in $6 \mathrm{MW}$ at 6 months of assessment, similar to that reported by Herridge.

Leone et al. in a cohort of 55 subjects with chest trauma and ICU admission reported $23 \%$ of patients with decreased FVC, 16\% with decreased FEV1/FVC ratio, and $71 \%$ of patients with a decreased DLCO $63 \%$ predicted at 6 months follow-up after chest trauma and ICU admission ${ }^{12}$. Deterioration in all PFTs evaluated was observed, suggesting that these subjects may show restrictive, obstructive, or mixed patterns associated with this type of trauma. Furthermore, although our group of subjects had a lower percentage of ARDS than that reported by Leone, the local and systemic inflammatory response secondary to traumatic lung injury and limiting factors in chest mobility such as pain, muscle weakness, diaphragm, and pleural damage may influence the different pulmonary function measurements at 3 and 6 months of assessment.

In addition to the severity of organ damage, pain management and physical rehabilitation are determining factors in the recovery of pulmonary function. Han et al. in a series of 80 subjects with a history of chest trauma found a $36 \mathrm{ml}$ (374 to 410 ) recovery in VC
( $p<0.001$ ) within the first $7-30$ days after trauma in subjects with adequate pain management ${ }^{16}$. Herridge et al. reported improvement with a rehabilitation program. Comprehensive medical management is necessary in these patients, as it may possibly be associated with a decrease in recovery times with improved physical deconditioning. Subjects with VC values of $<1.4$ I require longer inpatient and outpatient recovery time ${ }^{9}$. However, recovery times in rehabilitation programs for optimal improvement after lung injury are not known with certainty and individualized management and planning for each patient is very important.

The distance covered in the 6MW by subjects with a history of chest trauma shows statistically significant improvements at 6 months, possibly associated with the fact that the longer the assessment time and physical rehabilitation, the greater the distance covered in the test. Similar studies report a stepwise increase of $281 \mathrm{~m}$ at 3 months, $422 \mathrm{~m}$ at 12 months, and $436 \mathrm{~m}$ at 5 years of follow-up in patients with a history of trauma and ARDS ${ }^{14,15}$. Hanning et al. in a series of 13 subjects with a history of pulmonary contusion observed no alteration in VO2 at 12-48 months after trauma. However, 
the number of subjects included means that conclusions should be evaluated with caution ${ }^{17}$. We found a significant decrease in $\mathrm{VO} 2 / \mathrm{kg}$ in our younger population compared to this study ${ }^{17}$, possibly due to a greater involvement of trauma in our patients.

In subjects older than 55 years with a history of trauma, age was a factor related to longer ICU stay: $p=0.096$ (15 \pm 30 vs. $3 \pm 2$ days) and hospitalization $p=0.008$ (10 \pm 18 vs. $6 \pm 8$ days) when compared to population younger than 35 years. This suggests that age and its comorbidities are associated with worse outcomes and greater physical limitation ${ }^{18}$. Although the average age of combat-related casualties was lower than that found by Roth et al., hospital and ICU stays were prolonged due to the severity of injuries and the development of health care-associated infections or ARDS.

The weaknesses of our study are the small sample size and the loss of 14 subjects at 6 months follow-up. However, with the subjects admitted, we obtained a power of $81.8 \%$ for differences in pulmonary function. In addition, patients were relatively young and the impact of comorbidities on pulmonary function was unknown. The strengths of the present study are having a control group of subjects that allow us to compare the specific impact of chest trauma on pulmonary outcomes. It is necessary to generate studies that evaluate the impact of different pulmonary rehabilitation programs and processes that integrate multidisciplinary groups to optimize management guidelines in these patients, with a larger sample size and study time of more than 6 months to determine the evolution of pulmonary function in the population.

\section{Conclusion}

Subjects with combat-related chest trauma showed a decrease in FVC, FVC/FVC1, FEV1, VC, DLCO, and VO2 at 3 and 6 months of assessment. Complications associated with chest trauma management, pain management, and rehabilitation may be associated with recovery in these patients.

\section{Conflicts of interest}

The authors declare that there are no conflicts of interest.

\section{Funding}

Funding received from Hospital Militar Central.

\section{Ethical disclosures}

Protection of human and animal subjects. The authors declare that no experiments were performed on humans or animals for this study.

Confidentiality of data. The authors declare that they have followed the protocols of their work center on the publication of patient data.

Right to privacy and informed consent. The authors have obtained the written informed consent of the patients or subjects mentioned in the article. The corresponding author is in possession of this document.

\section{References}

1. Ivey KM, White CE, Wallum TE, Aden JK, Cannon JW, Chung KK, et al. Thoracic injuries in US combat casualties: a 10-year review of Operation Enduring Freedom and Iraqi Freedom. J Trauma Acute Care Surg. 2012;73 Suppl 5:S514-9.

2. Keneally R, Szpisjak D. Thoracic trauma in Iraq and Afghanistan. J Trauma Acute Care Surg. 2013;74:1292-7.

3. Dogrul BN, Kiliccalan I, Asci ES, Peker SC. Blunt trauma related chest wall and pulmonary injuries: an overview. Chin J Traumatol. 2020;23: 125-38.

4. Broderick SR. Hemothorax: etiology, diagnosis, and management. Thorac Surg Clin. 2013;23:89-96, vi-vii.

5. Drobin D, Gryth D, Persson JK, Rocksén D, Arborelius UP, Olsson LG, et al. Electroencephalogram, circulation, and lung function after high-velocity behind armor blunt trauma. J Trauma. 2007;63:405-13.

6. Desselle WJ, Greenhaw JJ, Trenthem LL, Fabian TC, Proctor KG. Macrophage cyclooxygenase expression, immunosuppression, and cardiopulmonary dysfunction after blunt chest trauma. J Trauma. 2001;51:239-51; discussion 251-2.

7. Stewart RM, Corneille MG. Common complications following thoracic trauma: their prevention and treatment. Semin Thorac Cardiovasc Surg. 2008;20:69-71.

8. Park PK, Cannon JW, Ye W, Blackbourne LH, Holcomb JB, Beninati W, et al. Incidence, risk factors, and mortality associated with acute respiratory distress syndrome in combat casualty care. J Trauma Acute Care Surg. 2016;81 Suppl 2:S150-6.

9. Bakhos C, O'Connor J, Kyriakides T, Abou-Nukta F, Bonadies J. Vital capacity as a predictor of outcome in elderly patients with rib fractures. J Trauma. 2006;61:131-4.

10. Hanna WC, Ferri LE, Fata P, Razek T, Mulder DS. The current status of traumatic diaphragmatic injury: lessons learned from 105 patients over 13 years. Ann Thorac Surg. 2008;85:1044-8.

11. Mayberry JC, Kroeker AD, Ham LB, Mullins RJ, Trunkey DD. Long-term morbidity, pain, and disability after repair of severe chest wall injuries. Am Surg. 2009;75:389-94.

12. Leone M, Brégeon F, Antonini F, Chaumoître K, Charvet $A$, Ban LH, et al. Long-term outcome in chest trauma. Anesthesiology. 2008;109: 864-71.

13. Weisgerber M, Danduran M, Meurer J, Hartmann K, Berger S, Flores G. Evaluation of Cooper 12-minute walk/run test as a marker of cardiorespiratory fitness in young urban children with persistent asthma. Clin $\mathrm{J}$ Sport Med. 2009;19:300-5.

14. Herridge MS, Cheung AM, Tansey CM, Matte-Martyn A, Diaz-Granados N, Al-Saidi $F$, et al. One-year outcomes in survivors of the acute respiratory distress syndrome. N Engl J Med. 2003;348:683-93.

15. Herridge MS, Tansey CM, Matté A, Tomlinson G, Diaz-Granados N, Cooper A, et al. Functional disability 5 years after acute respiratory distress syndrome. N Engl J Med. 2011;364:1293-304.

16. Han S, Baldemir M, Kose SK, Erdem D, Sakinci U. The time course of recovery following mild thoracic trauma. Heart Lung Circ. 2005;14:252-4.

17. Hanning CD, Ledingham E, Ledingham IM. Late respiratory sequelae of blunt chest injury: a preliminary report. Thorax. 1981;36:204-7.

18. Roth BJ, Velmahos GC, Oder DB, Vassiliu P, Tatevossian R, Demetriades $D$, et al. Penetrating trauma in patients older than 55 years: a case-control study. Injury. 2001;32:551-4. 